\title{
Reduced Carbon Monoxide Saturation Coverage on Vicinal Palladium Surfaces: the Importance of the Adsorption Site
}

\author{
Fernando Garcia-Martinez, Elisabeth Dietze, Frederik Schiller, Dorotea Gajdek, Lindsay R. Merte, \\ Sabrina M. Gericke, Johan Zetterberg, Stefano Albertin, Edvin Lundgren, Henrik Grönbeck, \\ and J. Enrique Ortega*
}

Cite This: J. Phys. Chem. Lett. 2021, 12, 9508-9515

Read Online

ACCESS | Lلll Metrics \& More | 回 Article Recommendations | st Supporting Information

ABSTRACT: Steps at metal surfaces may influence energetics and kinetics of catalytic reactions in unexpected ways. Here, we report a significant reduction of the $\mathrm{CO}$ saturation coverage in Pd vicinal surfaces, which in turn is relevant for the light-off of the CO oxidation reaction. The study is based on a systematic investigation of $\mathrm{CO}$ adsorption on vicinal $\mathrm{Pd}(111)$ surfaces making use of a curved Pd crystal. A combined X-ray Photoelectron Spectroscopy and DFT analysis allows us to demonstrate that an entire row of atomic sites under Pd steps remains free of $\mathrm{CO}$ upon saturation at $300 \mathrm{~K}$, leading to a step-densitydependent reduction of $\mathrm{CO}$ coverage that correlates with the observed decrease of the light-off temperature during $\mathrm{CO}$ oxidation in vicinal $\mathrm{Pd}$ surfaces.

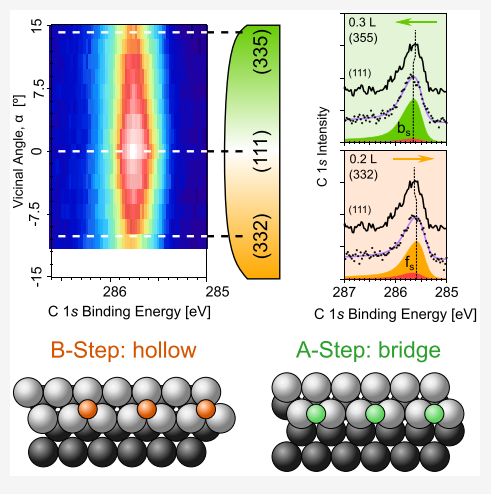

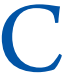
arbon monoxide (CO) adsorption on transition-metal surfaces is one of the elementary steps in the water-gas shift reaction, the Fischer-Tropsch synthesis, and the CO oxidation. $^{1-3} \mathrm{CO}$ oxidation is the canonical example of a Langmuir-Himshelwood process, in which the reactants, $\mathrm{CO}$ and $\mathrm{O}$, interact at neighboring surface sites and eventually form $\mathrm{CO}_{2} \cdot{ }^{2,3}$ In the presence of $\mathrm{CO}$ and $\mathrm{O}_{2}$ in the gas phase, coadsorption of $\mathrm{CO}$ and $\mathrm{O}$ is prevented at low temperatures, since $\mathrm{CO}$ saturates the surface and blocks the dissociative adsorption of $\mathrm{O}_{2}$. The $\mathrm{CO}$ saturating layer does not leave enough free surface space for $\mathrm{O}_{2}$ to impinge and react with coadsorbed CO. Thus, the temperature must be increased to facilitate $\mathrm{CO}$ desorption and creation of free surface sites for the reaction to start. ${ }^{4}$ Low temperature activity can be achieved by use of catalysts where the CO desorption energy is lowered, such as some bimetallic alloys, ${ }^{5}$ or bifunctional surfaces, such as oxide-supported nanoparticles, where $\mathrm{CO}$ and $\mathrm{O}_{2}$ adsorption occurs at physically separated nanoparticle and oxide sites. ${ }^{6,7}$

A vicinal metal surface may be viewed as a simple nanostructured catalyst, with different atomic coordination at terraces and steps, as well as a strong asymmetry in bonding configuration and lattice relaxation around the step edge. ${ }^{8}$ In the $\mathrm{CO}$ oxidation reaction, such a nanoscale structure of the vicinal surface is relevant since it affects the $\mathrm{CO}$ adsorption energy, which is higher at low coordination sites of steps as compared to the highly coordinated terraces. ${ }^{9}$ Therefore, close to the $\mathrm{CO}$ desorption temperature, one may expect COdepleted terraces, where molecular oxygen adsorbs and reacts with $\mathrm{CO}$ adsorbed at steps. ${ }^{10}$ However, at a high step density, the simple terrace/step picture breaks down, because lowercoordination steps and higher-coordination corner-row atoms effectively involve a larger portion of the surface. The question that arises is how does this peculiar structural asymmetry of vicinal surfaces influence $\mathrm{CO}$ chemisorption in densely stepped planes, such as to affect the activation of the catalytic $\mathrm{CO}$ oxidation.

Here, we investigate the impact of atomic steps on the adsorption of $\mathrm{CO}$ on $\mathrm{Pd}(111)$, by monitoring the sequence of adsorption sites during $\mathrm{CO}$ uptake at vicinal surfaces at $300 \mathrm{~K}$. To gain such information, we perform a full exploration of a curved Pd crystal sample using X-ray Photoelectron Spectroscopy (XPS) and Density Functional Theory (DFT). The Pd curved sample contains the (111) direction in the center of the surface and A- and B-type vicinal planes of increasing vicinal angle $\alpha$ at each side. Such a sequence of vicinal surfaces is probed with XPS (C 1s) after CO saturation at $300 \mathrm{~K}$, showing the surprising behavior displayed in Figure 1a. The CO saturation coverage in Pd steadily decreases from the (111) center $(\alpha=0)$ to the densely stepped sample edges by up to

Received: August 11, 2021

Accepted: September 22, 2021

Published: September 24, 2021 

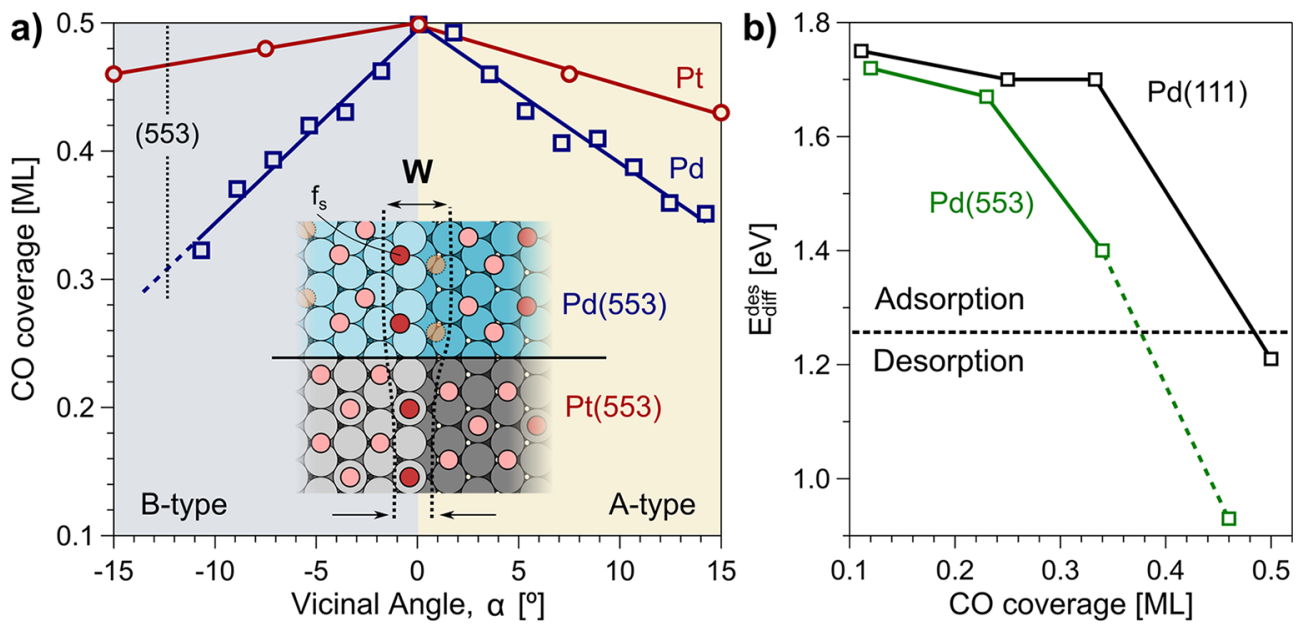

Figure 1. (a) CO saturation coverage at $300 \mathrm{~K}$ on $\mathrm{Pd}$ (blue) and Pt (red, ref 13.) vicinal surfaces, defined by the vicinal angle $\alpha$ with respect to (111). Lines are fit to data. The inset depicts the characteristic CO adsorption sites around monatomic B steps and marks the effective step width $(W)$ during $\mathrm{CO}$ saturation for both Pt $($ smaller $W$ ) and Pd (larger $W)$. (b) DFT-calculated differential desorption energy of CO, $E_{\text {diff }}$, as a function of coverage for $\mathrm{Pd}(553)$ and $\mathrm{Pd}(111)$. The horizontal dotted line marks the energy at which the desorption and adsorption rates are equal at $300 \mathrm{~K}$ and $10^{-9}$ mbar pressure.

$30-40 \%$, in contrast with the smaller $8-10 \%$ decrease found for the analogous curved $\mathrm{Pt}(111)$ surface. Our DFT-assisted analysis of the XPS spectra explains the Pd case: the lower (7fold) coordination of step atoms leads to preferential CO adsorption in the upper part of the step [ $f_{s}$ in Figure $\left.1 a\right]$, followed by filling of terrace sites (9-fold coordination), up to the Pd corner-row in the lower part of the next step [11-fold for $\mathrm{Pd}(553)]$, which remains empty. This results in a linear decrease of the $\mathrm{CO}$ saturation coverage as a function of the step density, which correlates with the observed reduction in light-off temperature across Pd vicinal surfaces. ${ }^{11,12}$

Our curved sample is a cylindrical section $\left(\Delta \alpha=30^{\circ}\right)$ of a Pd single crystal, referred to as c-Pd(111), described in detail in ref 12 and in the Supporting Information (SI, Figure S1). It features the (111) surface at the center, the (553) plane (Btype $\{111\}$ microfacet, $\left.\alpha=-12.3^{\circ}\right)$ close to one edge of the sample, and the (335) surface (A-type $\{100\}$ microfacet, $\alpha=$ $\left.+14.4^{\circ}\right)$ at the other edge. $\mathrm{Ar}^{+}$sputtering, $\mathrm{O}_{2}$ annealing, and high-temperature flashes were employed for cleaning the sample. The cleanliness and surface structure of the sample were checked by Low Energy Electron Diffraction (LEED) and XPS prior to the CO dosing experiments. The LEED pattern reveals a smoothly variable density of monatomic steps away from the (111) direction. ${ }^{11}$

XPS experiments were carried out at the FlexPES beamlime of MaxIV synchrotron in Lund, Sweden. A photon energy of $400 \mathrm{eV}$ was used to acquire C 1s spectra along the (111) normal, with the sample temperature kept at $300 \mathrm{~K}$. DoniacSunjić lines ${ }^{14}$ convoluted with a Gaussian profile and a Shirleytype ${ }^{15}$ background were employed in the fitting procedure. As reported in the literature, ${ }^{12,16,17}$ two vibrational excitations of the adsorbed $\mathrm{CO}$ molecules were considered for each of the CO species included in the fitting routine (see section S2 of the SI for the peak deconvolution protocol). To support the core-level analysis, Density Function Theory (DFT) calculations were performed with the Vienna Ab-initio Simulation Package (VASP) implementation, ${ }^{18-20}$ using the $\mathrm{PBE}^{21}$ exchange-correlation functional and the projector-augmented wave (PAW) method ${ }^{22,23}$ to describe the interaction between the valence electrons and the core. The calculations use
$\operatorname{Pd}(221)$ and $\operatorname{Pd}(112)$ as models for B- and A-types of steps, respectively. In addition, we use $\operatorname{Pd}(553)$ to study a surface with a large terrace and $\mathrm{Pd}(111)$ as a reference. Computational details and a full account of theoretical results are given in section S3 of the SI text, Tables S1-S5, and Figures S3-S5.

Room-temperature saturation is achieved after exposing the c-Pd(111) surface to a 10 Langmuir (L) CO dose at $300 \mathrm{~K}$. The sample is thereafter vertically moved in front of the X-ray beam in discrete steps, corresponding to $\Delta \alpha=1.75^{\circ}$, and the $\mathrm{C} 1 \mathrm{~s}$ spectrum is acquired. The photoelectron intensity (area under the peak) is converted into coverage assuming the saturation of the (111) plane with 0.5 monolayers (ML), which is in turn cross-checked through its characteristic LEED pattern. ${ }^{24}$ The CO coverage variation across the c-Pd(111) saturated surface is shown in Figure la, compared to the corresponding variation on its analogous c- $\mathrm{Pt}(111)$ crystal. $^{13,25}$ Lines are fit to data points and demonstrate that the total $\mathrm{CO}$ coverage decreases linearly as a function of the vicinal angle $\alpha$, that is, the step density. Away from the (111) plane, the CO saturation coverage slightly decreases for platinum, while it strikingly drops in palladium, up to $30-40 \%$, near the (335)(332) vicinal planes of the $A-B$ sides of the sample, respectively.

At fixed temperature, the $\mathrm{CO}$ saturation coverage is directly determined by the differential desorption energy, which depends on the CO-Pd bond strength and the (mainly) surface-mediated $\mathrm{CO}-\mathrm{CO}$ repulsive interaction strength. Assuming a nonactivated desorption process (no desorption barrier), the desorption energy is taken to be equal to the converse of the adsorption energy. Across the stepped surfaces, the $\mathrm{CO}$ desorption energy is higher above the steps and decreases across the terrace toward the next step (see Table S3 in SI). The repulsive $\mathrm{CO}-\mathrm{CO}$ interaction lowers the desorption energy dramatically in the dense layers, as reflected in Figure 1b. Here, we explore the coverage dependent desorption energy $\left(E_{\text {diff }}^{\text {des }}\right)$ for the $\operatorname{Pd}(553)$ plane (see S3 section in SI for more details). The coverage in the calculations is stepwise increased by adding one $\mathrm{CO}$ molecule per $2 \times 1$ row along the (110) direction, starting from $\mathrm{CO}$ adsorbed at hollow fcc sites in the step edge $\left(f_{s}, 0.11 \mathrm{ML}\right)$, followed by 

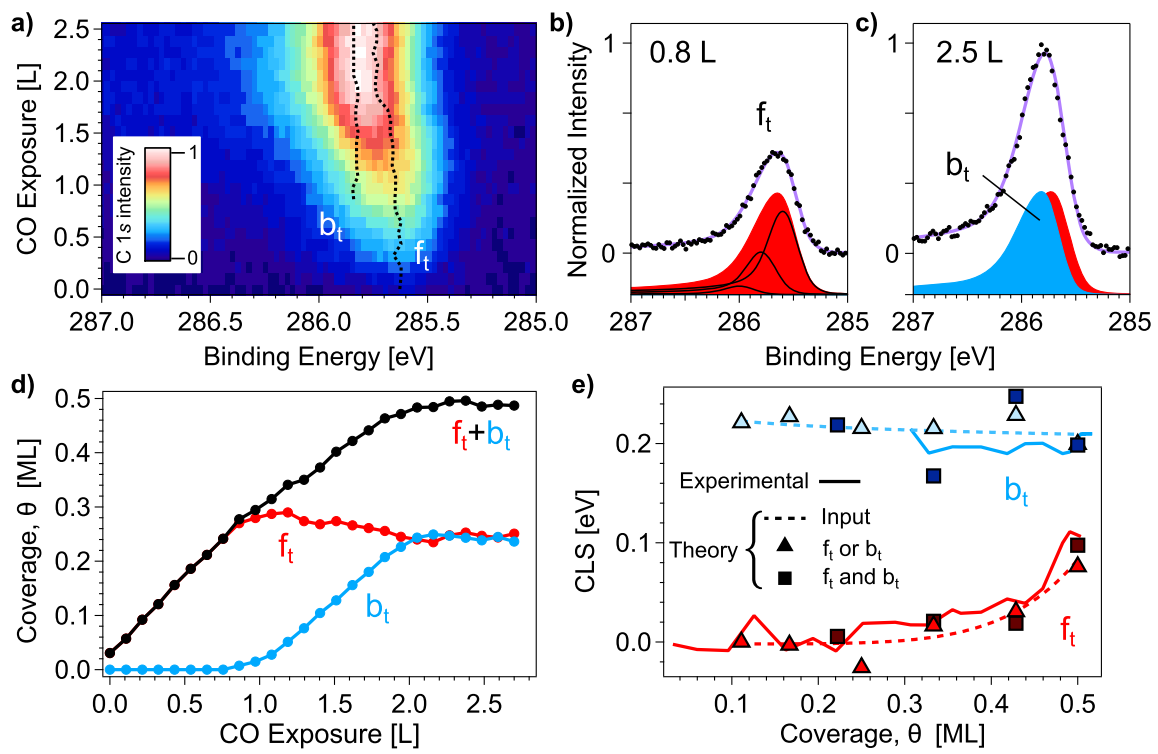

f)

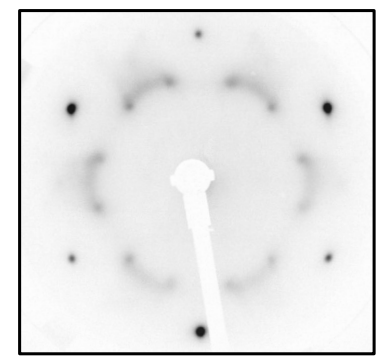

g)

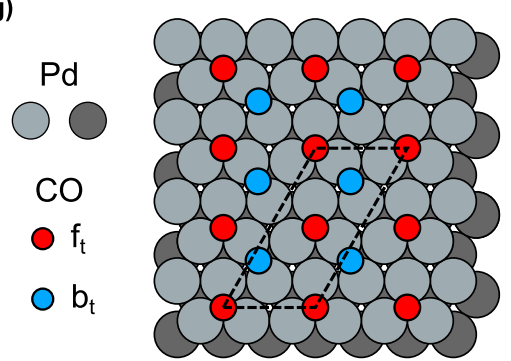

Figure 2. (a) C 1s intensity during CO uptake at the $\mathrm{Pd}(111)$ surface, acquired under $1 \times 10^{-9} \mathrm{mbar}$ CO dosage at $300 \mathrm{~K}$. Photon energy is 400 eV. $(b, c)$ Selected C 1s spectra at low $(0.8 \mathrm{~L} \mathrm{CO})$ and high $(2.5 \mathrm{~L})$ CO exposures, together with fitting lines for $\mathrm{f}_{\mathrm{t}}($ red $)$ and $b_{t}(\mathrm{blue})$ components. Solid lines correspond to main and double satellite contributions, assumed for all peaks. $(d) f_{t}, b_{t}$ and total-CO $\left(f_{t}+b_{t}\right)$ coverage evolution during the uptake shown in a. (e) Theoretical (squares, triangles) and experimental (solid lines) CLSs for $f_{t}$ and $b_{t}$ relative to the initial position of $f_{t}$ in the (111) surface. Dotted lines are smooth fits to theory data. (f) LEED pattern from $10 \mathrm{~L} \mathrm{CO}$ at Pd(111) (electron energy $80 \mathrm{eV}$ ). (g) Model proposed for $0.5 \mathrm{ML} \mathrm{CO}$ saturation of $\mathrm{Pd}(111)$ at $300 \mathrm{~K}$.

alternating bridge $\left(b_{t}\right)$ and $f c c\left(f_{t}\right)$ rows inside terraces, up to a fourth $\mathrm{CO} \mathrm{b}_{\mathrm{cr}}$ in the corner-row $(0.46 \mathrm{ML})$. Filling the (553) surface with the corner-row makes the differential desorption energy fall below $1.0 \mathrm{eV}$ (black dashed line), at which the $\mathrm{CO}$ adsorption rate is equal to the $\mathrm{CO}$ desorption rate at $T=300$ $\mathrm{K}$ and $10^{-9}$ mbar pressure. Therefore, the corner row is empty in $\operatorname{Pd}(553)$, and the $300 \mathrm{~K}$ saturation coverage corresponds to three $\mathrm{CO}$ rows per terrace (nominally $0.34 \mathrm{ML}$ ). Note that the coordination number of corner-row surface atoms is 11 , which implies that the $\mathrm{CO}$ desorption energy is reduced with respect to the (111) surface also in the absence of $\mathrm{CO}-\mathrm{CO}$ repulsion. In contrast to the $\mathrm{Pd}(553)$ case, the desorption energy at $\operatorname{Pd}(111)$ is above the line for adsorption/desorption equilibrium close to $0.5 \mathrm{ML} \mathrm{CO}$, demonstrating the difference in saturation coverage. On the other hand, atop and bridge adsorption configurations on the analogous $\mathrm{Pt}(553)$ stepped surface [see Figure 1a] allow higher saturation coverage without involving the highly coordinated corner-row atoms. This is a consequence of the spatial extension of the atop adsorption (one surface atom) as compared to the fcc adsorption (three surface atoms). We may define the effective step width $W$ sketched in Figure 1a, as the relative surface area affected by $\mathrm{CO}$ adsorption at step sites in a vicinal surface, which is larger in Pd than in Pt. As shown below, experiments on the curved surface allows us to estimate $W$ quantitatively.
A more detailed look into the $\mathrm{C} 1 \mathrm{~s}$ spectrum during $\mathrm{CO}$ uptake at $300 \mathrm{~K}$ allows us to asses the sequence of $\mathrm{CO}$ bonding sites at different planes, and then postulate $\mathrm{CO}$ saturation geometries. For uptake experiments, the clean sample was exposed to $1 \times 10^{-9}$ mbar $\mathrm{CO}$ while continuously monitoring the $\mathrm{C} 1 \mathrm{~s}$ region. It is essential to first investigate the CO adsorption on the (111) plane, whose rich family of structural arrangements as a function of coverage and temperature has been a matter of discussion. ${ }^{16,17,24,26-31}$ Of particular relevance is the $0.5 \mathrm{ML}$ saturation at $300 \mathrm{~K},{ }^{16,24}$ which corresponds to the coverage at which $\mathrm{O}_{2}$ can not adsorb even at near-ambient pressures. ${ }^{12}$ As shown in Figure 2a, at the lowest CO dose a single peak is detected at $285.62 \mathrm{eV}$ [2b)], which we assign to $\mathrm{CO}$ adsorbed at hollow fcc sites at terraces $\left(f_{t}\right){ }^{16} f_{t}$ steadily grows with $\mathrm{CO}$ exposure up to $1.2 \mathrm{~L}$, accompanied by a smooth shift to larger binding energy. At this point, and following Surnev et al., ${ }^{16}$ the changing shape of the peak and its shift reveals the emergence of the $b_{t}$ contribution (adsorption in bridge sites at terraces) at $285.77 \mathrm{eV}$.

Note that $f_{t}$ and $b_{t}$ peaks in Figure $2 c$ are close and difficult to resolve. On the other hand, when the $\mathrm{CO}$ coverage approaches the $0.5 \mathrm{ML}$ saturation, the mutual $\mathrm{CO}-\mathrm{CO}$ repulsion increases (see Table S3 in SI). Thus, shifts in corelevel binding energies are expected as they reflect the state of bonding. ${ }^{32}$ To obtain a reliable uptake curve [Figure $2 \mathrm{~d}$ ], we need to assist the line-fit analysis through the Core-Level Shift 

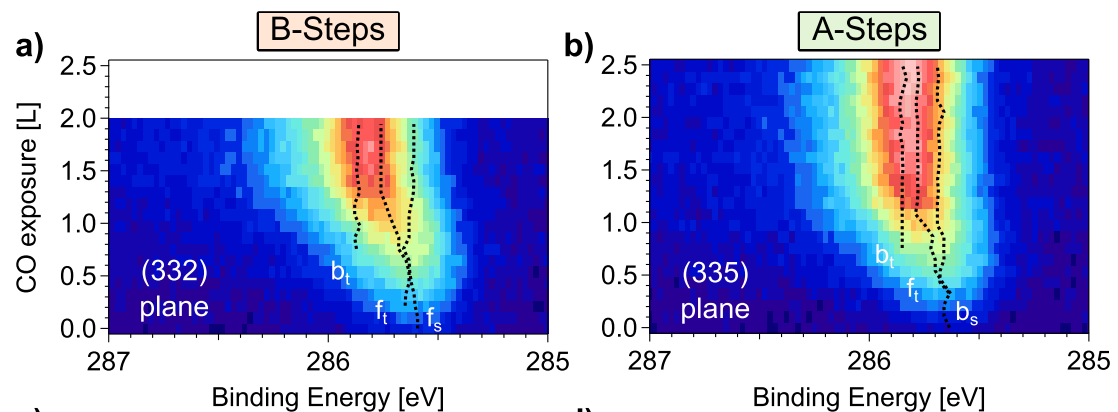

c)
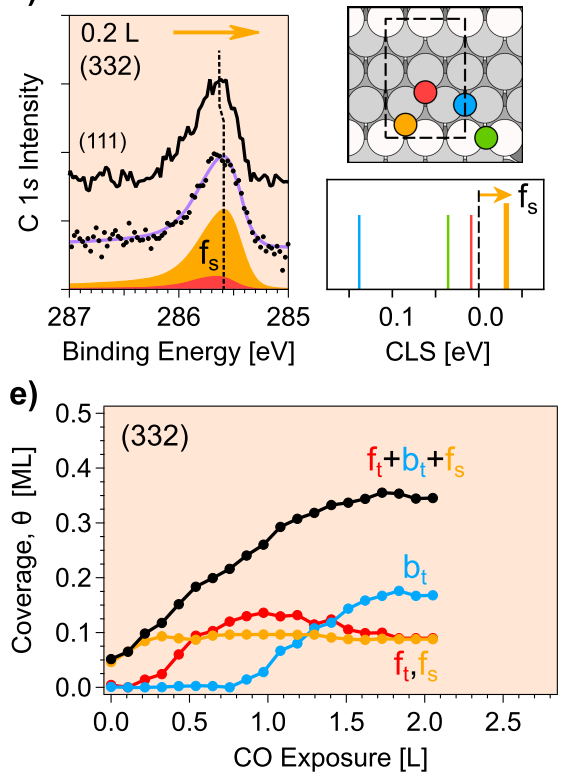

$0.1 \quad 0.0$ CLS [eV] d)

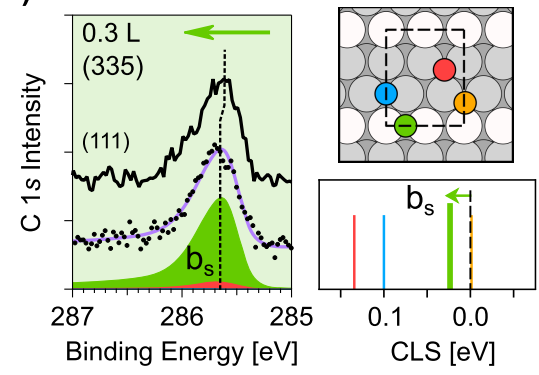

f)

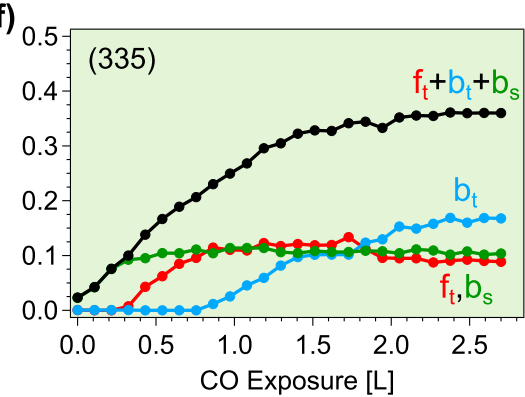

Figure 3. (a,b) C $1 s$ intensity during CO uptake at the (332) and (335) surfaces, acquired under $1 \times 10^{-9} \mathrm{mbar} \mathrm{CO}$ at $300 \mathrm{~K}$. Photon energy is 400 $\mathrm{eV}$ and color scale is the same as in Figure 2. (c,d) Low CO dose spectra, with line fitting, for each of the aforementioned surfaces, compared to the same dose in $\mathrm{Pd}(111)$. The right side in each case shows the $\mathrm{C}$ 1s core-level shift (CLS) calculation, with respect to a single $\mathrm{f}_{\mathrm{t}}$ molecule on the (111) surface, for $\mathrm{CO}$ on $\mathrm{Pd}(221)$ and $\mathrm{Pd}(112)$, respectively, in the sketched configurations. (e,f) CO coverage evolution during the CO uptake at the (332) and (335) surfaces, respectively.

(CLS) DFT calculations shown in Figure 2e (symbols, see section S3 in SI for details). The calculations reveal that $f_{t}$ and $b_{t}$ do not shift equally as a function of coverage. Using an averaged theoretical curve (dotted line) as initial input to fit the experimental core levels, we obtain the solid lines, which represent experimental CLSs relative to the initial position of $f_{t}$. These lines also appear overlaid in the color plots of Figure 2a. $\mathrm{f}_{\mathrm{t}}$ shifts slightly by approximately $+0.1 \mathrm{eV}$ from $0.34 \mathrm{ML}$ (corresponding to the $\left(3^{1 / 2} \times 3^{1 / 2}\right) \mathrm{R} 30^{\circ}$ structure $^{16}$ ) to saturation, while $b_{t}$ remains virtually unchanged. The respective peak intensities, converted to $\mathrm{CO}$ coverage by normalizing to $0.5 \mathrm{ML}$ at saturation, are represented in Figure 2d. Notably, this DFT-assisted fitting procedure leads to equal $50 \%$ contribution of $b_{t}$ and $f_{t}$ sites, in contrast to a larger $b_{t}$ weight if the binding energy shift correction is not applied.

The structure of the CO-saturated (111) surface was probed with LEED. The $0.5 \mathrm{ML}$ pattern shown in Figure $2 \mathrm{f}$ agrees with that of Ertl and Koch, ${ }^{24}$ although it appears better defined in our case. It has been argued that this pattern progressively "splits" from the $\left(3^{1 / 2} \times 3^{1 / 2}\right) \mathrm{R} 30^{\circ}$ structure, due to the increasing presence of disordered domains from $1 / 3$ to 0.5 ML. ${ }^{16,32}$ However, assuming the equivalent 0.25:0.25 ML $b_{t} / f_{t}$ coverage, we postulate the more simple $c(4 \times 2)-4 \mathrm{CO}$ arrangement sketched in Figure $2 \mathrm{~g}$. As discussed in section S2 in the SI, this structure directly produces most of the extinctions needed to generate the main spots of Figure $2 \mathrm{~g}$ out of the fundamental $c(4 \times 2)$ pattern. Nevertheless, to explain the diffuse arc-shaped intensity without altering the $b_{t} / f_{t}$ ratio, there is a need to consider a certain degree of disorder, induced by the large $\mathrm{CO}$ mobility at $300 \mathrm{~K}$, and also the presence of phase and antiphase $c(4 \times 2)-4 \mathrm{CO}$ domains (see section S2 and Figure S2 in SI).

The combination of XPS and DFT is powerful to extract detailed information from $\mathrm{CO}$ uptake curves at stepped surfaces. As an example, in Figure 3 we investigate the $\mathrm{CO}$ uptake at the B-type (332) and the A-type (335) surfaces of the c-Pd(111) sample. Color plots in Figure 3a,b correspond to $\mathrm{C} 1 \mathrm{~s}$ spectra acquired in separate uptake experiments on both surfaces. Immediately after leaking $\mathrm{CO}$ into the chamber, a peak emerges at 285.66 and $285.60 \mathrm{eV}$ in the (332) and (335) planes, respectively. As in other vicinal metal surfaces, $^{33-36}$ we ascribe such an emerging line to $\mathrm{CO}$ adsorbed at steps. Although very slightly, peak energies are shifted toward lower (negative shift) and higher (positive shift) binding energy with respect to the $f_{t}$ peak in the (111) plane at similar CO exposures, as shown in the left side panels of Figure $3 \mathrm{c}, \mathrm{d}$, respectively. The opposite shifts reflect a different adsorption site at the step edge, as revealed by the DFT calculation sketched in the respective right side panels. Here, we consider separately different $\mathrm{CO}$ adsorption sites at 
a)

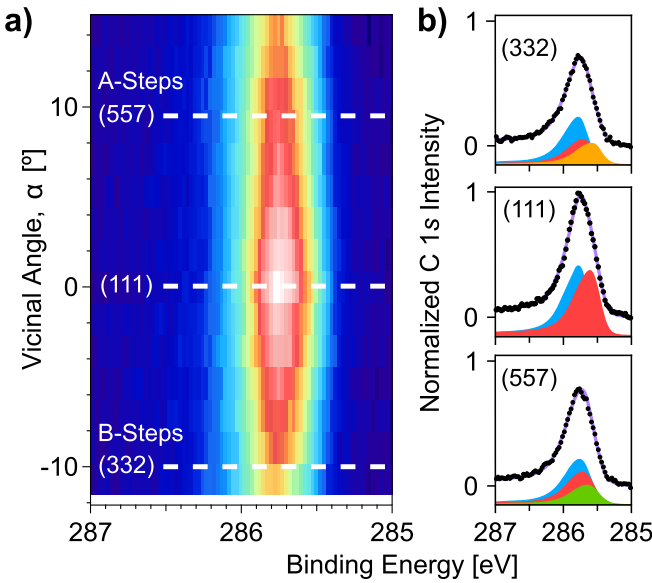

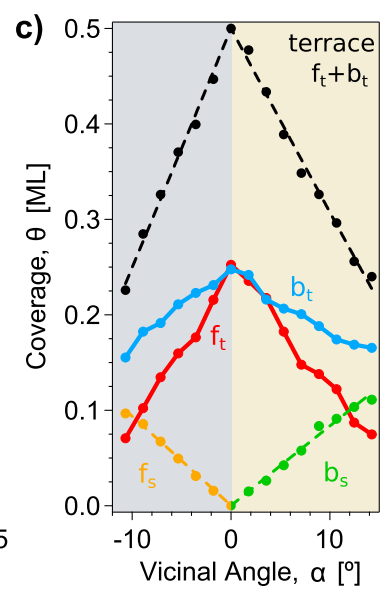

d)

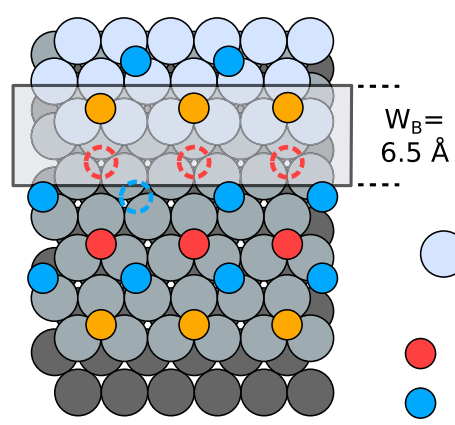

e)

(557), $\alpha=+9.5^{\circ}$

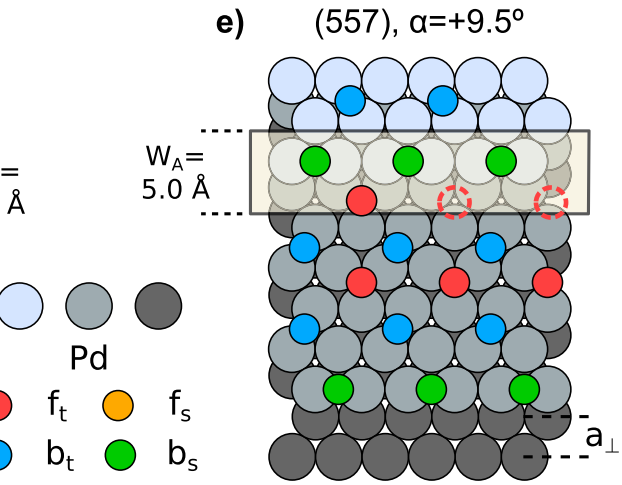

Figure 4. (a) C 1s $\alpha$-scan across the CO-saturated c-Pd(111) surface, acquired after exposing the clean sample to $10 \mathrm{~L}$ of $\mathrm{CO}$ at $300 \mathrm{~K}$. Photon energy and color scale as in Figure 2. (b) Individual spectra for relevant surfaces with the respective line-fitted components: terrace fcc-hollow $\left(f_{t}\right.$, red) and bridge $\left(b_{t}\right.$, blue) and step fcc-hollow $\left(f_{s}\right.$, orange) and bridge $\left(b_{s}\right.$, green). (c) Coverage of different CO species as a function of $\alpha$. Black markers correspond to terrace-CO $\left(\mathrm{f}_{\mathrm{t}}+\mathrm{b}_{\mathrm{t}}\right)$. The black dashed line fits terrace-CO data to the $W$ model described in ref 39 and in section S1 of the SI. (d,e) CO layer saturation models proposed for the (332) and (557) surfaces. Dotted circles denote missing CO. The color code is the same as in $b$ and $c$.

$\operatorname{Pd}(221)$ and $\operatorname{Pd}(112)$ surfaces, which are used to represent Band A-type vicinal surfaces, respectively. All terrace sites exhibit positive shifts with respect to $f_{t}$ on $\operatorname{Pd}(111)$, including stepbridge sites $\left(b_{s}\right)$. The hollow fcc site of the step $\left(f_{s}\right)$ has no shift on the A-type step, whereas the shift is negative on the Btype step. We therefore conclude that $\mathrm{CO}$ adsorbs at $\mathrm{f}_{\mathrm{s}}$ step sites on the B-type $\operatorname{Pd}(332)$ surface and, as previously observed, ${ }^{37,38}$ at $b_{s}$ sites on the A-type $\operatorname{Pd}(335)$.

At a higher CO dose, terrace $f_{t}$ and $b_{t}$ peaks emerge, leading to major changes in the $\mathrm{C}$ 1s features of Figure 3a,b. A minimal three-peak $\left(f_{t}, b_{t}\right.$ and step) fit is thus required, which can reliably be done thanks to the curved surface systematics. The resulting binding energies for the three peaks are marked with dotted lines in Figure 3a,b, while the intensity (coverage) variation is shown in Figure 3e,f. Terrace and step peaks on both (332) and (335) surfaces evolve in a similar way. On the B-type (332) surface, the $\mathrm{f}_{\mathrm{s}}$ step species saturates $(0.5 \mathrm{CO} / \mathrm{step}$ atom, $0.094 \mathrm{ML}$ ) after exposure to $0.4 \mathrm{~L} \mathrm{CO}$. Above this dose, a second feature ascribed to $f_{t}$ emerges. As observed on the (111) surface, $b_{t}$ starts to grow when $f_{t}$ reaches its maximum at $1.0 \mathrm{~L}$, and $f_{t}$ shifts toward larger binding energy as the system approaches saturation. At this point, both $f_{t}$ and $f_{s}$ have similar intensities, which is about half of the $b_{t}$ intensity. On the Atype (335) surface, the $b_{s}$ step peak saturates $(0.5 \mathrm{CO} /$ step atom, $0.088 \mathrm{ML}$ ) at $0.3 \mathrm{~L}$, where the $\mathrm{f}_{\mathrm{t}}$ emerges and grows up to its maximum at $0.8 \mathrm{~L}$. From here, $b_{t}$ grows and $f_{t}$ slightly attenuates. On both (332) and (335) surfaces, the evolution of terrace $f_{t}$ and $b_{t}$ species is very similar to that observed in the (111) surface, although the relative intensity of $b_{t}$ at saturation is significantly larger. This latter reflects the absence of $\mathrm{CO}$ at $\mathrm{f}_{\mathrm{cr}}$ sites in the corner row, as discussed below.

Having identified the variety of the $\mathrm{CO}$ adsorption sites at the characteristic (332) and (335) vicinal planes, next we investigate the $\alpha$-dependent evolution of each species in the CO-saturated c-Pd(111) surface. Figure 4a shows such an $\alpha$ scan as a color plot. After exposing the clean sample to $10 \mathrm{~L}$ of $\mathrm{CO}$ at $300 \mathrm{~K}$, individual $\mathrm{C} 1 \mathrm{~s}$ spectra were systematically acquired at 15 different points on the curved surface. The plot nicely reflects the marked decrease of the CO layer coverage from the (111) center and toward the sample edges, represented in Figure 1a. All individual spectra are consistently fitted, following the analysis discussed in the previous section. As an example, we present spectra and fitting lines at three different sample points in Figure $4 \mathrm{~b}$. The resulting intensity variation of each component is shown in Figure 4c, together with the total "terrace-CO" $\left(\mathrm{f}_{\mathrm{t}}+\mathrm{b}_{\mathrm{t}}\right)$ signal (black markers). At $\alpha=0$, i.e., the (111) plane, $\mathrm{f}_{\mathrm{t}}$ and $\mathrm{b}_{\mathrm{t}}$ equally occupy the surface. Both decrease almost linearly as a function of $\alpha$, as expected from the effective reduction of the occupied terrace area, due to both step and unoccupied corner-row Pd atoms. It is worth noting that $b_{t}$ decreases at a lower rate as compared to $f_{t}$, leading to the significant $f_{t} / b_{t}$ unbalance at the stepped edges, discussed in Figure 3. In contrast to terrace-CO, the intensity from $f_{s}$ and $b_{s}$ step species increases linearly away from the 
(111) center, and approximately at the same rate at A and B steps. This consistent step-peak intensity growth demonstrates that the number of $\mathrm{CO}$ molecules per step atom is not affected by the step density or type in this case.

To asses the effective width of the step $W$, that is, the surface area affected by $\mathrm{CO}$ adsorption at steps [see Figure 1a], we fit the total terrace- $\mathrm{CO}$ and step-CO intensities using the $W$ model described in ref 39 and section S1 of the SI. Assuming monatomic steps (2.25 $\AA$ height) and a fixed terrace-CO density of $0.5 \mathrm{ML}$, the model renders the effective step width $W_{\mathrm{A}}=6.5 \AA$ and $W_{\mathrm{B}}=5.0 \AA$, for $\mathrm{A}$ and $\mathrm{B}$ steps. It also yields $\Theta_{\mathrm{S}, \mathrm{A}}=0.39$ and $\Theta_{\mathrm{S}, \mathrm{B}}=0.44$ molecules per step atom, close to the expected 0.5 ratio. $W_{\mathrm{A}}$ and $W_{\mathrm{B}}$ can be compared to the distance between Pd atomic rows $\left(a_{\perp}, 2.75 \AA\right)$, revealing that the effective width of A- and B-type steps exceeds, by $136 \%$ and $82 \%$, that of the atom row $a_{\perp}$, respectively. Therefore, CO occupation of step sites not only affects the step-atom row but also blocks $\mathrm{CO}$ adsorption within a significant portion of the terrace, namely the corner row. As discussed above, CO adsorption at bridge or hollow positions in the corner-row leads to a large increase in the intermolecular repulsion, and hence corner-row $\mathrm{Pd}$ atoms remain $\mathrm{CO}$ free.

In Figure $4 \mathrm{~d}, \mathrm{e}$, we postulate the $\mathrm{CO}$ layer structure at saturation for characteristic B-type and A-type vicinal $\mathrm{Pd}$ planes, namely, $\operatorname{Pd}(332)$ and $\operatorname{Pd}(557)$, respectively. In essence, we first complete the 0.5 molecule-per-atom step edge and then fill up alternating $b_{t}$ (blue) and $f_{t}$ (red) rows as we move into the terrace. Following Figure $3 \mathrm{e}, \mathrm{f}$ results, the filling process begins with $f_{s}$ (orange) or $b_{s}$ (green) sites at the (332) or (557) plane, respectively, and extends to the terrace until the total (experimental) saturation coverage is reached in each case, $0.34 \mathrm{ML}$ for $\mathrm{Pd}(332)$ and $0.40 \mathrm{ML}$ for $\mathrm{Pd}(557)$. On the basis of the theoretical analysis of Figure 1b, the entire row of fcc hollow sites in the corner-row remains empty in the (332) surface, while in the (557) surface three out of four such corner-row sites are unoccupied. Interestingly, such a small difference in corner-row occupation at A- and B-type steps could also be expected from their distinct atomic coordination and the small adsorption energy difference that it implies: on A-type surfaces, such as $\operatorname{Pd}(557)$, the hollow site at the corner row involves $\mathrm{CO}$ bonding to a 10 -fold coordinated $\mathrm{Pd}$ atom. The resulting CO desorption energy is therefore slightly higher than the one expected at the B-type $\mathrm{Pd}(332)$ surface, where corner-row $\mathrm{Pd}$ atoms are 11 -fold coordinated.

In summary, vicinal surfaces possess asymmetric bonding configurations around steps, with metal atoms having lower and higher coordination at step and corner atoms, respectively, as compared to terrace atoms. Here, we have investigated how this property alters $\mathrm{CO}$ adsorption, particularly at densely stepped surfaces. Our XPS intensity profile of a curved $\mathrm{Pd}(111)$ surface saturated with $\mathrm{CO}$ at $300 \mathrm{~K}$ demonstrates that Pd vicinal surfaces exhibit a significant reduction of the saturation coverage as a function of the step density, which DFT calculations link with the effective elimination of the $\mathrm{CO}$ adsorbed at corner-row atoms. This nonuniform coverage scenario is confirmed by the analysis of the $\mathrm{C} 1 \mathrm{~s}$ core level during $\mathrm{CO}$ uptake at $300 \mathrm{~K}$, performed at different vicinal planes of the curved Pd substrate. With the assistance of DFT calculations, which facilitate the identification of the different core-level contributions, $\mathrm{CO}$ uptake curves reveal that the characteristic 1:1 bridge/hollow balance of the (111) surface is broken at terraces in vicinal surfaces, owing to empty unfavorable hollow sites at the corner row. A point-by-point analysis of the saturation spectra at the curved surface confirms the steady reduction of terrace hollow sites with respect to bridge sites as a function of the step density in both A- and Btype vicinal surfaces.

\section{ASSOCIATED CONTENT}

\section{Supporting Information}

The Supporting Information is available free of charge at https://pubs.acs.org/doi/10.1021/acs.jpclett.1c02639.

Description of the $\mathrm{Pd}$ curved sample used in the experiments; description of the $W$ model to estimate the effective step width; line fit and peak deconvolution protocol; LEED simulations for several 0.5 ML structures of $\mathrm{CO}$ chemisorbed on $\mathrm{Pd} ; \mathrm{CO}$ adsorption on Pd; CO desorption; C 1s core-level shift; (Figure S1) sketch of the curved Pd sample; (Figure S2) $0.5 \mathrm{ML}$ structures and calculated LEED patterns; (Table 1) $\mathrm{CO} / \mathrm{Pd}$ surface models for DFT calculations; (Table 2) adsorption energies at different configurations; (Table 3) coverage dependent mean adsorption energies; (Table 4) C 1s core-level shift for different $\mathrm{CO}$ coverage on $\mathrm{Pd}(111)$; (Table 5) C 1s core-level shift at low CO coverage on $\operatorname{Pd}(112)$ and $\operatorname{Pd}(221)$; (Figure S3) adsorption rates for $\mathrm{CO}$ on $\mathrm{Pd}(111)$ at $300 \mathrm{~K}$; (Figure S4) adsorption sites on the (111), (112), (221), (553), and (332) surfaces; (Figure S5) sketch for the C 1s corelevel shift calculation; graph for the $\mathrm{C} 1 \mathrm{~s}$ core-level shift versus adsorption energy (PDF)

\section{AUTHOR INFORMATION}

\section{Corresponding Author}

J. Enrique Ortega - Centro de Física de Materiales CSIC/ UPV-EHU-Materials Physics Center, 20018 San Sebastian, Spain; Departamento Física Aplicada, Universidad del País Vasco, 20018 San Sebastian, Spain; Donostia International Physics Centre, 20018 San Sebastian, Spain; orcid.org/ 0000-0002-6643-806X; Email: enrique.ortega@ehu.es

\section{Authors}

Fernando Garcia-Martinez - Centro de Física de Materiales CSIC/UPV-EHU-Materials Physics Center, 20018 San Sebastian, Spain

Elisabeth Dietze - Department of Physics and Competence Centre for Catalysis, Chalmers University of Technology, 41296 Göteborg, Sweden; 이이이.org/0000-0001-96199242

Frederik Schiller - Centro de Física de Materiales CSIC/ UPV-EHU-Materials Physics Center, 20018 San Sebastian, Spain; o orcid.org/0000-0003-1727-3542

Dorotea Gajdek - Department of Materials Science and Applied Mathematics, Malmö University, 21118 Malmö, Sweden

Lindsay R. Merte - Synchrotron Radiation Research, Lund University, 22100 Lund, Sweden; Department of Materials Science and Applied Mathematics, Malmö University, 21118 Malmö, Sweden; ○ orcid.org/0000-0002-3213-4199

Sabrina M. Gericke - Combustion Physics, Lund University, 22100 Lund, Sweden

Johan Zetterberg - Combustion Physics, Lund University, 22100 Lund, Sweden; (1) orcid.org/0000-0002-0882-1482

Stefano Albertin - Synchrotron Radiation Research, Lund University, 22100 Lund, Sweden 
Edvin Lundgren - Synchrotron Radiation Research, Lund University, 22100 Lund, Sweden; (1) orcid.org/0000-00023692-6142

Henrik Grönbeck - Department of Physics and Competence Centre for Catalysis, Chalmers University of Technology, 41296 Göteborg, Sweden; 이이. orcid/0000-0002-87092889

Complete contact information is available at:

https://pubs.acs.org/10.1021/acs.jpclett.1c02639

\section{Notes}

The authors declare no competing financial interest.

\section{ACKNOWLEDGMENTS}

We acknowledge financial support from the Spanish Ministry of Science and Innovation (Grants MAT-2017-88374-P, PID2019-107338RB-C63, PID2020-116093RB-C44), the Basque Government (Grants IT-1255-19), the Knut and Alice Wallenberg foundation (DNR KAW 2015.0058 "Atomistic Design of new Catalysts"), and the Swedish Research Council (DNR 349-2011-6491 "Catalysis on the Atomic Scale"). Financial support is acknowledged from the Swedish Research Council (2016-5234). The DFT calculations were performed at C3SE (Göteborg) via a SNIC grant. We acknowledge MAX IV Laboratory support, in particular Alexei Preobrajenski, for their help in synchrotron experiments, which were carried out at the FlexPES beamline under Proposal 20190717. Research conducted at MAX IV, a Swedish national user facility, is supported by the Swedish Research council under contract 2018-07152, the Swedish Governmental Agency for Innovation Systems under contract 2018-04969, and Formas under contract 2019-02496. Moreover, the research leading to this result has been supported by the project CALIPSOplus under the Grant Agreement 730872 from the EU Framework Programme for Research and Innovation HORIZON 2020.

\section{REFERENCES}

(1) Jahangiri, H.; Bennett, J.; Mahjoubi, P.; Wilson, K.; Gu, S. A review of advanced catalyst development for Fischer-Tropsch synthesis of hydrocarbons from biomass derived syn-gas. Catal. Sci. Technol. 2014, 4, 2210-2229.

(2) Ertl, G. Reactions at well-defined surfaces. Surf. Sci. 1994, 299$300,742-754$.

(3) Ertl, G. Reactions at Solid Surfaces; John Wiley \& Sons, Inc.: Hoboken, NJ, 2009; Chapter 6, pp 123-157.

(4) Gustafson, J.; Balmes, O.; Zhang, C.; Shipilin, M.; Schaefer, A.; Hagman, B.; Merte, L. R.; Martin, N. M.; Carlsson, P.-A.; Jankowski, M.; et al. The Role of Oxides in Catalytic $\mathrm{CO}$ Oxidation over Rhodium and Palladium. ACS Catal. 2018, 8, 4438-4445.

(5) Gonzalez, S.; Illas, F. CO adsorption on monometallic Pd, Rh, $\mathrm{Cu}$ and bimetallic $\mathrm{PdCu}$ and $\mathrm{RhCu}$ monolayers supported on $\mathrm{Ru}(0001)$. Surf. Sci. 2005, 598, 144-155.

(6) Vayssilov, G. N.; Lykhach, Y.; Migani, A.; Staudt, T.; Petrova, G. P.; Tsud, N.; Skála, T.; Bruix, A.; Illas, F.; Prince, K. C.; et al. Support nanostructure boosts oxygen transfer to catalytically active platinum nanoparticles. Nat. Mater. 2011, 10, 310-315.

(7) Liu, X.; Tang, Y.; Shen, M.; Li, W.; Chu, S.; Shan, B.; Chen, R. Bifunctional CO oxidation over Mn-mullite anchored $\mathrm{Pt}$ subnanoclusters via atomic layer deposition. Chemical Science 2018, 9, 2469-2473.

(8) Prévot, G.; Girard, Y.; Repain, V.; Rousset, S.; Coati, A.; Garreau, Y.; Paul, J.; Mammen, N.; Narasimhan, S. Elastic displacements and step interactions on metallic surfaces: Grazingincidence x-ray diffraction and ab initio study of $\mathrm{Au}(332)$. Phys. Rev. B: Condens. Matter Mater. Phys. 2010, 81, 1-10.
(9) Vollmer, S.; Witte, G.; Wöll, C. Determination of site specific adsorption energies of CO on copper. Catal. Lett. 2001, 77, 97-101.

(10) Jørgensen, M.; Grönbeck, H. The Site-Assembly Determines Catalytic Activity of Nanoparticles. Angew. Chem., Int. Ed. 2018, 57, 5086-5089.

(11) Blomberg, S.; Zetterberg, J.; Zhou, J.; Merte, L. R.; Gustafson, J.; Shipilin, M.; Trinchero, A.; Miccio, L. A.; Magaña, A.; Ilyn, M.; et al. Strain Dependent Light-off Temperature in Catalysis Revealed by Planar Laser-Induced Fluorescence. ACS Catal. 2017, 7, 110-114.

(12) Schiller, F.; Ilyn, M.; Pérez-Dieste, V.; Escudero, C.; HuckIriart, C.; Ruiz del Arbol, N.; Hagman, B.; Merte, L. R.; Bertram, F.; Shipilin, M.; et al. Catalytic Oxidation of Carbon Monoxide on a Curved Pd Crystal: Spatial Variation of Active and Poisoning Phases in Stationary Conditions. J. Am. Chem. Soc. 2018, 140, 16245-16252.

(13) Garcia-Martinez, F.; García-Fernández, C.; Simonovis, J. P.; Hunt, A.; Walter, A.; Waluyo, I.; Bertram, F.; Merte, L. R.; Shipilin, M.; Pfaff, S.; et al. Catalytic Oxidation of $\mathrm{CO}$ on a Curved $\mathrm{Pt}(111)$ Surface: Simultaneous Ignition at All Facets through a Transient COO Complex. Angew. Chem., Int. Ed. 2020, 59, 20037-20043.

(14) Doniach, S.; Sunjic, M. Many-electron singularity i n x-ray photoemission andx-ray line spectra from metals. J. Phys. C: Solid State Phys. 1970, 3, 285-291.

(15) Shirley, D. A. High-resolution x-ray photoemission spectrum of the valence bands of gold. Phys. Rev. B 1972, 5, 4709-4714.

(16) Surnev, S.; Sock, M.; Ramsey, M.; Netzer, F.; Wiklund, M.; Borg, M.; Andersen, J. CO adsorption on $\mathrm{Pd}(111)$ : a high-resolution core level photoemission and electron energy loss spectroscopy study. Surf. Sci. 2000, 470, 171-185.

(17) Martin, N. M.; Van den Bossche, M.; Grönbeck, H.; Hakanoglu, C.; Zhang, F.; Li, T.; Gustafson, J.; Weaver, J. F.; Lundgren, E. CO Adsorption on Clean and Oxidized Pd(111). J. Phys. Chem. C 2014, 118, 1118-1128.

(18) Kresse, G.; Hafner, J. Ab Initio Molecular Dynamics for Liquid Metals. Phys. Rev. B: Condens. Matter Mater. Phys. 1993, 47, 558-561.

(19) Kresse, G.; Hafner, J. Ab Initio Molecular-Dynamics Simulation of the Liquid-Metal-Amorphous-Semiconductor Transition in Germanium. Phys. Rev. B: Condens. Matter Mater. Phys. 1994, 49, 14251-14269.

(20) Kresse, G.; Furthmüller, J. Efficient Iterative Schemes for Ab Initio Total-Energy Calculations using a Plane-Wave Basis Set. Phys. Rev. B: Condens. Matter Mater. Phys. 1996, 54, 11169-11186.

(21) Perdew, J. P.; Burke, K.; Ernzerhof, M. Generalized Gradient Approximation Made Simple. Phys. Rev. Lett. 1997, 78, 1396.

(22) Blöchl, P. E. Projector augmented-wave method. Phys. Rev. B: Condens. Matter Mater. Phys. 1994, 50, 17953-17979.

(23) Kresse, G.; Joubert, D. From Ultrasoft Pseudopotentials to the Projector Augmented-Wave Method. Phys. Rev. B: Condens. Matter Mater. Phys. 1999, 59, 1758-1775.

(24) Ertl, G.; Koch, J. Adsorption von CO auf einer Palladium(111)Oberfläche. Z. Naturforsch., A: Phys. Sci. 1970, 25, 1906-1912.

(25) Walter, A. L.; Schiller, F.; Corso, M.; Merte, L. R.; Bertram, F.; Lobo-Checa, J.; Shipilin, M.; Gustafson, J.; Lundgren, E.; Brión-Ríos, A. X.; et al. X-ray photoemission analysis of clean and carbon monoxide-chemisorbed platinum(111) stepped surfaces using a curved crystal. Nat. Commun. 2015, 6, 8903.

(26) Conrad, H.; Ertl, G.; Koch, J.; Latta, E. E. Adsorption of CO on Pd single crystal surfaces. Surf. Sci. 1974, 43, 462-480.

(27) Biberrian, J. P.; Van Hove, M. A. A new model for CO ordering at high coverages on low index metal surfaces: A correlation between LEED, HREELS and IRS. II. CO adsorbed on fcc (111) and hep (0001) surfaces. Surf. Sci. 1984, 138, 361-389.

(28) Ohtani, H.; Hove, M. A.; Somorjai, G. A. Leed intensity analysis of the surface structures of $\mathrm{Pd}(111)$ and of $\mathrm{CO}$ adsorbed on $\operatorname{Pd}(111)$ in a $(\sqrt{3} \times \sqrt{3}) \mathrm{R} 30^{\circ}$ arrangement. Surf. Sci. 1987, 187, 372-386.

(29) Gießel, T.; Schaff, O.; Hirschmugl, C. J.; Fernandez, V.; Schindler, K. M.; Theobald, A.; Bao, S.; Lindsay, R.; Berndt, W.; Bradshaw, A. M.; et al. A photoelectron diffraction study of ordered 
structures in the chemisorption system Pd $\{111\}-$ CO. Surf. Sci. 1998, 406, 90-102.

(30) Rose, M. K.; Mitsui, T.; Dunphy, J.; Borg, A.; Ogletree, D. F.; Salmeron, M.; Sautet, P. Ordered structures of CO on $\operatorname{Pd}\left(\begin{array}{lll}1 & 1 & 1\end{array}\right)$ studied by STM. Surf. Sci. 2002, 512, 48-60.

(31) Hooshmand, Z.; Le, D.; Rahman, T. S. CO adsorption on $\operatorname{Pd}(111)$ at 0.5 ML: A first principles study. Surf. Sci. 2017, 655, 711.

(32) Surnev, S.; Sock, M.; Ramsey, M.; Netzer, F.; Wiklund, M.; Borg, M.; Andersen, J. CO adsorption on $\mathrm{Pd}(111)$ : a high-resolution core level photoemission and electron energy loss spectroscopy study. Surf. Sci. 2000, 470, 171-185.

(33) Tränkenschuh, B.; Papp, C.; Fuhrmann, T.; Denecke, R.; Steinrück, H. P. The dissimilar twins - a comparative, site-selective in situ study of $\mathrm{CO}$ adsorption and desorption on $\mathrm{Pt}(322)$ and $\mathrm{Pt}(355)$. Surf. Sci. 2007, 601, 1108-1117.

(34) Tränkenschuh, B.; Fritsche, N.; Fuhrmann, T.; Papp, C.; Zhu, J. F.; Denecke, R.; Steinrück, H. P. A site-selective in situ study of CO adsorption and desorption on $\mathrm{Pt}(355)$. J. Chem. Phys. 2006, 124, 074712.

(35) Stroppa, A.; Mittendorfer, F.; Andersen, J. N.; Parteder, G.; Allegretti, F.; Surnev, S.; Netzer, F. P. Adsorption and dissociation of $\mathrm{CO}$ on bare and Ni-decorated stepped $\mathrm{Rh}(553)$ Surfaces. J. Phys. Chem. C 2009, 113, 942-949.

(36) Sinniah, K.; Dorsett, H. E.; Reutt-Robey, J. E. Chemisorption on stepped metal surfaces: CO/vicinal $\mathrm{Ni}(100)$. J. Chem. Phys. 1993, 98, 9018-9029.

(37) Wille, A.; Nickut, P.; Al-Shamery, K. Low temperature dissociation of $\mathrm{CO}$ at reactive step sites of $\mathrm{Pd}(112)$. J. Mol. Struct. 2004, 695-696, 345-352.

(38) Zhang, J.; Zhang, X.; Wang, Z.; Diao, Z. Adsorption of carbon monoxide on $\operatorname{Pd}(311)$ and (211) surfaces. Appl. Surf. Sci. 2008, 254, 6327-6331.

(39) Garcia-Martinez, F.; Schiller, F.; Blomberg, S.; Shipilin, M.; Merte, L. R.; Gustafson, J.; Lundgren, E.; Ortega, J. E. CO chemisorption on vicinal $\mathrm{Rh}(111)$ surfaces studied with a curved crystal. J. Phys. Chem. C 2020, 124, 9305-9313. 